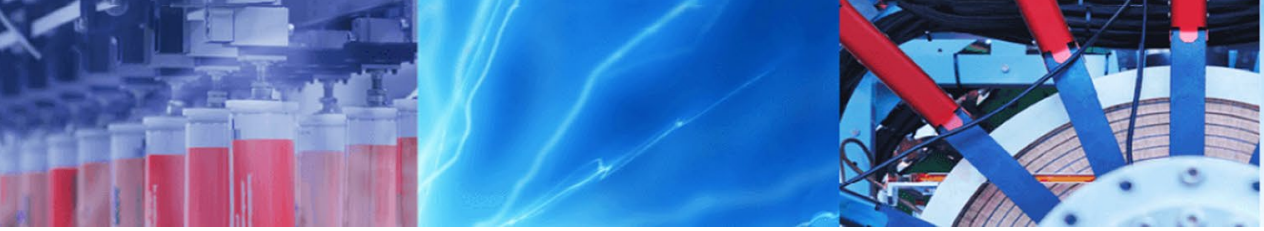

Research Article

\title{
Orthonormal, moment preserving boundary wavelet scaling functions in Python
}

\author{
Josefine Holm ${ }^{1}$ (D) . Thomas Arildsen ${ }^{2}$ (D) . Morten Nielsen ${ }^{1}$ (1) . Steffen Lønsmann Nielsen ${ }^{1}$
}

Received: 27 April 2020 / Accepted: 6 November 2020 / Published online: 20 November 2020

(c) Springer Nature Switzerland AG 2020

\begin{abstract}
In this paper we derive an orthonormal basis of wavelet scaling functions for $L^{2}([0,1])$ motivated by the need for such a basis in the field of generalized sampling. A special property of this basis is that it includes carefully constructed boundary functions and it can be constructed with arbitrary smoothness. This construction makes assumptions about the signal outside the interval unnecessary. Furthermore, we provide a Python package implementing this wavelet decomposition. Wavelets defined on a bounded interval are widely used for signal analysis, compression, and for numerical solution of differential equations. We show that for many cases using the basis that we derive results in smaller error than the commonly used alternative.
\end{abstract}

Keywords Wavelet · Boundary functions · Software Package · Fourier · Generalized Sampling $\cdot$ Python

\section{Introduction}

Wavelets have been a very successful tool in signal processing since the early 1990 s, se e.g. $[7,17,23]$. Recently they have been used extensively in the new field of generalized sampling $[1,2,10,14]$. Generalized sampling enables the transform of a sampled signal from one basis to another. A prominent example of the use of generalized sampling is magnetic resonance imaging (MRI). In the case of MRI, the hardware dictates the sampling setup and Fourier samples are naturally obtained. Images are known to be well represented with wavelets but not in a Fourier basis [22]. Therefore it is desirable to map the Fourier samples to wavelet coefficients using the technique of generalized sampling. In this paper, we derive an orthonomal moment preserving wavelet basis on an interval and the Fourier transform of this basis, which is well adapted for use in generalized sampling algorithms such as described in [10]. For an efficient implementation of generalized sampling, it is essential to have closed form expressions for the wavelets as well as for their Fourier transforms. We will use the construction of boundary functions, which was introduced in [5] and studied further in [3, §4], to obtain such representations. Other applications include lossy compression of finite signals and to the study of classic boundary problems, for which the alternative is to extend the signal beyond the given interval. However the alternative approach is heavily dependent on the choice of the particular extension.

First we will discuss in some detail the construction of the boundary functions. Based on this construction we derive the Fourier transform of the boundary functions. Due to Plancherel's theorem there exists a matrix which can orthonormalize the boundary functions in both domains. We will carefully construct such a matrix based on $[3$, p. 10]. Then we will show an example of the construction for Daubechies 2. Finally we will compare the

$\square$ Josefine Holm, jho@es.aau.dk; Thomas Arildsen, tari@its.aau.dk; Morten Nielsen, mnielsen@math.aau.dk|'Department of Mathematical Sciences, Aalborg University, Aalborg, Denmark. ${ }^{2}$ Department of Electronic Systems, Aalborg University, Aalborg, Denmark. 
derived method to the classic method of mirrored extenstion, in the context of compression.

Accompanying this paper is an implementation of the described boundary functions in Python 3, [13]. The package can be used to create orthonormal, moment preserving boundary wavelet scaling functions for a wide range of wavelets such as Daubechies wavelets, symlets and coiflets. All examples given in this paper can be reproduced with the accompanying test files.

\subsection{Relation to other work}

The theory presented in this paper elaborates work with wavelets on the interval done in the early nineties, [7, 17, 23], in order to facilitate the implementation in Python. There are several existing packages for a variety of programming languages, including Python and MATLAB, which deal with boundary effects. The most commonly used packages for wavelets, Python's PyWavelets and MATLAB's dwt, do not make use of boundary functions at all, instead the signal is artificially extended. Several types of extensions are available to get acceptable results depending on the type of signal. Orthonormal moment preserving boundary functions are available and are used for generalized sampling in MATLAB in [10] and in Julia in [14]. Both software packages use the filter coefficients presented in [6, Table 3 and 4]. The WaveLab function MakeCDJVFilter() for MATLAB only provides coefficients for Daubechies 2 and 3 and the Julia package from [14] includes coefficients for Daubechies 2 to 8 . In contrast, our package constructs boundary functions based on interior wavelet filter coefficients and can therefore be used for a much wider variety of wavelets, such as symlets and coiflets.

\section{Background and notation}

In this paper we will use standard compactly supported wavelets generated by a multi-resolution analysis, see $[4$, Definition 3.6.2], with $\psi$ the generating wavelet and $\phi$ the scaling function. Specifically, we consider an orthonormal basis of scaling functions for $L^{2}(\mathbb{R})$, and use it to construct an orthonormal basis of scaling functions for $L^{2}([0,1])$. We use the notation

$\phi_{J, k}(x)=2^{\frac{\jmath}{2}} \phi\left(2^{\lrcorner} x-k\right)$,

where $x \in \mathbb{R}, J \in \mathbb{Z}_{+}$and $k \in \mathbb{Z}$.

For most dyadic wavelets there is no closed form expression for the scaling function. It can, however, be approximated at specific points using the so-called cascade algorithm [19, Section 6.2]. The cascade algorithm is based on the two-scale equation $\phi(x)=\sqrt{2} \sum_{v \in \mathbb{Z}} h_{\nu} \phi(2 x-v)$.

Here $\left\{h_{v}\right\}_{v \in \mathbb{Z}}$ are filter coefficients and only $2 a-1$ of them are non-zero. Furthermore

$\sum_{v \in \mathbb{Z}} h_{v}=\sqrt{2}$

The number of vanishing moments $a$, for a function $f \in L^{2}(\mathbb{R})$, is the highest value of $a$ such that

$\int_{\mathbb{R}} x^{\prime} f(x) d x=0, \quad \forall I=0,1, \ldots, a-1$,

$[17$, eq. (7.69)]. A consequence of $[17$, Theorem 7.4$]$ is that a wavelet basis can reconstruct polynomials of a degree up to the number of vanishing moments for the wavelet minus one. This is a key feature for a wavelet basis; and by moment preserving boundary wavelet scaling functions we mean that the boundary functions preserve this property.

The Fourier transform is defined as

$\mathcal{F} f](\omega)=\int_{\mathbb{R}} f(x) e^{-2 \pi i \omega x} d x$.

For a general scaling function the following is true:

$\left.\mathcal{F}\left[\phi_{J, k}\right](\omega)=2^{-J / 2} \exp \left(-2 \pi i k 2^{-\jmath} \omega\right) \mathcal{F} \phi\right]\left(2^{-\jmath} \omega\right)$.

A general Daubechies scaling function, $\phi$, is defined by its

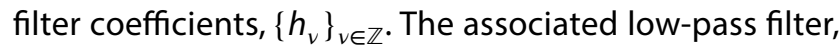
$m_{0}(\omega)$, is defined as

$m_{0}(\omega)=\sum_{\nu \in \mathbb{Z}} h_{\nu} \exp (-2 \pi i v \omega)$

and the Fourier transform can be computed as

$\mathcal{F} \phi](\omega)=\prod_{j=1}^{\infty} m_{0}\left(2^{-j} \omega\right)$,

where $m_{0}(0)=1$ to ensure convergence, $[6$, p. 54] .

\section{Derivation of boundary wavelet scaling functions}

In this section the construction of dyadic boundary wavelet scaling functions will be explored. Dyadic boundary functions were introduced in [5], see also [3, §4]. As noted in [15] this construction of wavelets on an interval is associated with a multiresolution analysis. Furthermore [12] argues that the construction can be extended to wavelet functions. 
All polynomials of degree less than or equal to $a-1$ can be written as a linear combination of $\left\{\phi_{J, k}\right\}_{k \in \mathbb{Z}}$ but when restricted to a closed interval, say $[0,1]$, this is no longer the case. To generate all polynomials up to degree $a-1$ we need to add boundary functions, equal to the number of vanishing moments, at each boundary.

It is desirable to have $2^{\jmath}$ scaling functions when working with $[0,1]$. If we use a wavelet with $a$ vanishing moments there are $2^{\jmath}-2 a+2$ interior functions for sufficiently large $J$. This leaves room for $a-1$ extra functions at each boundary which gives a system that can generate polynomials up to degree $a-2$. If we want degree $a-1$, like we have for the corresponding system on $(-\infty, \infty)$, we have to omit the two outermost interior functions to make room for extra boundary functions, [6, p. 70].

As mentioned in Sect. 2 , all monomials, $x^{\alpha}$ with coefficient $\alpha \leq a-1$, can be written as $x^{\alpha}=\sum_{k}\left\langle x^{\alpha}, \phi_{J, k}\right\rangle \phi_{J, k}(x)$. When restricted to $[0,1]$, we get

$\left.x^{\alpha}\right|_{[0,1]}=\left.\left(\sum_{k=-2 a+2}^{0}+\sum_{k=1}^{2^{\lrcorner}-2 a}+\sum_{k=2^{\lrcorner}-2 a+1}^{2^{\jmath}-1}\right)\left\langle x^{\alpha}, \phi_{J, k}\right\rangle \phi_{J, k}(x)\right|_{[0,1]}$.

Define

$$
\begin{aligned}
& X_{J, \alpha}^{L}=\left.\sum_{k=-2 a+2}^{0}\left\langle x^{\alpha}, \phi_{J, k}\right\rangle \phi_{J, k}(x)\right|_{[0,1]^{\prime}} \\
& X_{J, \alpha}^{R}=\left.\sum_{k=2^{J}-2 a+1}^{2^{J}-1}\left\langle x^{\alpha}, \phi_{J, k}\right\rangle \phi_{J, k}(x)\right|_{[0,1]},
\end{aligned}
$$

then

$$
\left.x^{\alpha}\right|_{[0,1]}=X_{J, \alpha}^{L}+\left.\sum_{k=1}^{2^{J}-2 a}\left\langle x^{\alpha}, \phi_{J, k}\right\rangle \phi_{J, k}(x)\right|_{[0,1]}+X_{J, \alpha}^{R}
$$

and $\left\{X_{J, \alpha}^{L}\right\}_{\alpha \leq a-1} \cup\left\{X_{J, \alpha}^{R}\right\}_{\alpha \leq a-1} \cup\left\{\left.\phi_{J, k}\right|_{[0,1]}\right\}_{k=1}^{2^{J}-2 a}$ forms a basis for $L^{2}([0,1])$. In order to evaluate $(10)$, the inner product $\left\langle x^{\alpha}, \phi_{J, k}\right\rangle$ needs to be rewritten in a form that can be evaluated numerically. This will be done in two main steps. First by making a change of variable in $\left\langle x^{\alpha}, \phi_{J, k}\right\rangle$, given by $u=2^{\jmath} x-k \Rightarrow x=2^{-\lrcorner}(u+k)$ and $d x=2^{-\jmath} d u$ we get:

$$
\begin{aligned}
\left\langle x^{\alpha}, \phi_{J, k}\right\rangle & =\int_{\mathbb{R}} x^{\alpha} 2^{J / 2} \phi\left(2^{J} x-k\right) d x \\
& =2^{-\jmath} 2^{\frac{\jmath}{2}} \int_{\mathbb{R}}\left(2^{-\jmath}(u+k)\right)^{\alpha} \phi(u) d u \\
& =2^{-\jmath+\frac{\jmath}{2}-J \alpha} \int_{\mathbb{R}}(u+k)^{\alpha} \phi(u) d u \\
& =2^{-\jmath+\frac{\jmath}{2}-J \alpha} \int_{\mathbb{R}} \sum_{l=0}^{\alpha}\left(\begin{array}{l}
\alpha \\
l
\end{array}\right) u^{l} k^{\alpha-l} \phi(u) d u \\
& =2^{-\jmath+\frac{\jmath}{2}-J \alpha} \sum_{l=0}^{\alpha}\left(\begin{array}{l}
\alpha \\
l
\end{array}\right) k^{\alpha-l} \int_{\mathbb{R}} u^{l} \phi(u) d u .
\end{aligned}
$$

Secondly, the quantity $\left\langle x^{\prime}, \phi\right\rangle$ is known as the moments of the scaling function. In [20, pp. 395-396] and [16, Section 5] a recursion relation for the moments is derived. For $I=0$ we have $\left\langle x^{0}, \phi\right\rangle=\int_{\mathbb{R}} \phi(x) d x=1$. For larger / we have

$$
\begin{aligned}
\left\langle x^{\prime}, \phi\right\rangle= & \int_{\mathbb{R}} x^{\prime} \phi(x) d x \\
= & \int_{\mathbb{R}} \frac{1}{\sqrt{2}}\left(\frac{x}{2}\right)^{\prime} \frac{1}{\sqrt{2}} \phi\left(\frac{x}{2}\right) d x \\
= & \frac{1}{2^{\prime} \sqrt{2}} \int_{\mathbb{R}} x^{\prime} \sum_{v \in \mathbb{Z}} h_{v} \phi(x-v) d x \\
= & \frac{1}{2^{\prime} \sqrt{2}} \sum_{v \in \mathbb{Z}} h_{v} \int_{\mathbb{R}}(v+v)^{\prime} \phi(v) d v \\
= & \frac{1}{2^{\prime} \sqrt{2}} \sum_{v \in \mathbb{Z}} h_{v} \sum_{m=0}^{l}\left(\begin{array}{c}
I \\
m
\end{array}\right) v^{I-m} \int_{\mathbb{R}} v^{m} \phi(v) d v \\
= & \frac{1}{2^{\prime} \sqrt{2}} \sum_{v \in \mathbb{Z}} h_{v} \sum_{m=0}^{I-1}\left(\begin{array}{c}
I \\
m
\end{array}\right) v^{I-m} \int_{\mathbb{R}} v^{m} \phi(v) d v \\
& +\frac{1}{2^{\prime} \sqrt{2}} \sum_{v \in \mathbb{Z}} h_{v} \int_{\mathbb{R}} v^{\prime} \phi(v) d v,
\end{aligned}
$$

where in the fourth equation we have made the change of variable $v=x-v \Rightarrow x=v+v$. From the above we obtain

$\int_{\mathbb{R}} v^{\prime} \phi(v) d v-\frac{1}{2^{\prime}} \int_{\mathbb{R}} v^{\prime} \phi(v) d v$

$=\frac{1}{2^{\prime} \sqrt{2}} \sum_{v \in \mathbb{Z}} h_{v} \sum_{m=0}^{I-1}\left(\begin{array}{c}l \\ m\end{array}\right) v^{I-m} \int_{\mathbb{R}} v^{m} \phi(v) d v$,

$\int_{\mathbb{R}} v^{\prime} \phi(v) d v$

$=\frac{1}{\left(2^{I}-1\right) \sqrt{2}} \sum_{v \in \mathbb{Z}} h_{v} \sum_{m=0}^{I-1}\left(\begin{array}{c}I \\ m\end{array}\right) v^{I-m} \int_{\mathbb{R}} v^{m} \phi(v) d v$.

With these two steps (10) can be evaluated numerically.

To obtain an orthogonal basis for $L^{2}([0,1])$, the $\left\{\phi_{J, \alpha^{\prime}}^{L} \phi_{J, \alpha}^{R}\right\}$ need to be orthogonalized. The 
orthogonalization will be handled in Sect. 3.2. They are already orthogonal to $\phi_{\lrcorner, m}$ and linearly independent.

\subsection{The frequency domain}

The left and right boundary functions can be written as a linear combination of $\left.\phi_{J, k}\right|_{[0,1]}, k=-2 a+2, \ldots, 0$ and $k=2^{\jmath}-2 a+1, \ldots, 2^{\jmath}-1$ respectively. And since the Fourier transform is linear, the Fourier transformed boundary functions are

$$
\begin{aligned}
& \mathcal{F}\left[X_{J, \alpha}^{L}\right]=\sum_{k=-2 a+2}^{0}\left\langle x^{\alpha}, \phi_{J, k}\right\rangle \mathcal{F}\left[\left.\phi_{J, k}(x)\right|_{[0,1]}\right], \\
& \mathcal{F}\left[X_{J, \alpha}^{R}\right]=\sum_{k=2^{J}-2 a+1}^{2^{J}-1}\left\langle x^{\alpha}, \phi_{J, k}\right\rangle \mathcal{F}\left[\left.\phi_{J, k}(x)\right|_{[0,1]}\right] .
\end{aligned}
$$

The inner products in the functions can be evaluated in the same way as in the previous section. The next thing to consider is $\mathcal{F}\left[\left.\phi_{J, k}(x)\right|_{[0,1]}\right]$ :

$\mathcal{F}\left[\left.\phi_{J, k}(x)\right|_{[0,1]}\right]=\mathcal{F}\left[\phi_{J, k}\right] * \mathcal{F}\left[\chi_{[0,1]}\right]$,

here $\chi_{[0,1]}$ is the indicator function and $*$ denotes the convolution. Furthermore, we know that

$$
\begin{aligned}
\mathcal{F}\left[\phi_{J, k}\right](\omega)= & \left.2^{\frac{-\jmath}{2}} \exp \left(2 \pi i k 2^{-\jmath} \omega\right) \mathcal{F} \phi\right]\left(2^{-\jmath} \omega\right) \\
= & 2^{\frac{-\jmath}{2}} \exp \left(2 \pi i k 2^{-\jmath} \omega\right) \prod_{l=1}^{\infty} m_{0}\left(2^{-l} 2^{-\jmath} \omega\right) \\
= & 2^{\frac{-\jmath}{2}} \exp \left(2 \pi i k 2^{-\jmath} \omega\right) \prod_{l=1}^{\infty} \sum_{v \in \mathbb{Z}} h_{v} \\
& \exp \left(-2 \pi i v 2^{-l} 2^{-\jmath} \omega\right)
\end{aligned}
$$

and

$\left.\mathcal{F} \chi_{[0,1]}\right](\omega)= \begin{cases}\frac{1-\exp (-2 \pi i \omega)}{2 \pi i \omega}, & \omega \neq 0, \\ 1, & \omega=0 .\end{cases}$

Due to Plancherel's theorem, [4, eq. 2.14], which states that the Fourier transform is norm-preserving, it does not matter if the boundary functions are orthogonalized before or after the transformation so we choose to do the latter.

\subsection{Orthogonalization}

We will orthogonalize the boundary functions using the procedure described in $[3$, p. 10]. Let us first consider the left boundary functions. Denote the unorthogonalized functions $X_{J, \alpha}^{L}$ and the orthogonal $\phi_{J, \alpha^{\prime}}^{L}$ then
$\phi_{J, \alpha}^{L}=\sum_{\beta=0}^{a-1} A^{\alpha \beta} X_{J, \beta}^{L}$

for some $a \times a$ matrix $A_{L}=A_{J, L}=\left\{A^{\alpha \beta}\right\}_{\alpha=0, \beta=0}^{a-1, a-1}$. For these functions to form an orthonormal set, they must fulfil

$\delta_{\alpha \beta}=\left\langle\phi_{J, \alpha^{\prime}}^{L} \phi_{J, \beta}^{L}\right\rangle_{[0,1]}=\sum_{\gamma, \eta=0}^{a-1} A_{L}^{\alpha \gamma} \bar{A}_{L}^{\beta \eta}\left\langle X_{J, \gamma^{\prime}}^{L}, X_{J, \eta}^{L}\right\rangle_{[0,1]}$.

Define the matrix $M_{L}=M_{J, L}=\left\{M^{\alpha \beta}\right\}_{\alpha=0, \beta=0}^{a-1, a-1}$ by

$M_{L}^{\alpha \beta}=\left\langle X_{J, \gamma}^{L}, X_{J, \eta}^{L}\right\rangle_{[0,1]^{\prime}}$

then (20) can be written as $I_{a \times a}=A_{L} M_{L} A_{L}^{*}$. It is possible to show that $M_{L}$ is symmetric and positive definite. Because of this it has a Cholesky decomposition, $M_{L}=C_{L} C_{L}^{*}$, which gives $A_{L}=C_{L}^{-1}$. Taking a closer look at $M_{L}$, we get that

$$
\begin{aligned}
M_{L}^{\alpha \beta} & =\left.\left.\int_{\mathbb{R}} \sum_{k=-2 a+2}^{0}\left\langle x^{\alpha}, \phi_{J, k}\right\rangle \phi_{J, k}(x)\right|_{[0,1]} \sum_{l=-2 a+2}^{0}\left\langle x^{\beta}, \phi_{J, l}\right\rangle \phi_{J, l}(x)\right|_{[0,1]} d x \\
& =\sum_{k=-2 a+2}^{0} \sum_{l=-2 a+2}^{0}\left\langle x^{\alpha}, \phi_{J, k}\right\rangle\left\langle x^{\beta}, \phi_{J, l}\right\rangle \int_{0}^{1} \phi_{J, k} \phi_{J, l} d x .
\end{aligned}
$$

The matrix $M_{L}$ 's dependency on $J$ makes $M_{L}$ ill-conditioned when $J$ is large. Here ill-conditioned means that the condition number for the matrix defined as $\kappa(A)=\|A\|_{2}\left\|A^{-1}\right\|_{2}$ is large, [21, (12.15)]. The integral is independent of $J$ for $J \geq a$ and the inner product has the following relation

$\left\langle x^{\alpha}, \phi_{J, k}\right\rangle=2^{-\frac{\jmath}{2}} 2^{-\jmath \alpha}\left\langle x^{\alpha}, \phi_{0, k}\right\rangle$

so we can factorize $M_{L}$ as $M_{L}=2^{-J} h M_{L}^{\prime} h$, where

$$
\begin{aligned}
M_{L}^{\prime} & =\sum_{k=-2 a+2}^{0} \sum_{l=-2 a+2}^{0}\left\langle x^{\alpha}, \phi_{0, k}\right\rangle\left\langle x^{\beta}, \phi_{0, I}\right\rangle \int_{0}^{1} \phi_{J, k} \phi_{J, l} d x, \\
h & =\operatorname{diag}\left(2^{-J \alpha}\right) \quad \alpha=0, \ldots, a-1 .
\end{aligned}
$$

From this we get

$I_{a \times a}=A_{L} M_{L} A_{L}^{*}=2^{-J} A_{L} h M_{L}^{\prime} h A_{L}^{*}=2^{-J} A_{L} h C_{L}^{\prime}\left(C_{L}^{\prime}\right)^{*} h A_{L}^{*}$

and thus

$A_{L}=2^{-\frac{\jmath}{2}}\left(C_{L}^{\prime}\right)^{-1} h^{-1}$.

The matrix $C_{L}^{\prime}$ has a constant relatively low condition number for all $J$, so the calculation of $A_{L}$ is stable. We do the same for the right boundary functions, but we have an additional dependency on $J$ because $k=\left\{2^{\lrcorner}-2 a+1, \ldots, 2^{\lrcorner}-1\right\}$. Substituting $k^{\prime}=2^{-\lrcorner} k$ lets us factor out part of the $J$-dependency from the inner product terms 


$$
\begin{aligned}
\left\langle x^{\alpha}, \phi_{0, k}\right\rangle & =\sum_{l=0}^{\alpha}\left(\begin{array}{l}
\alpha \\
l
\end{array}\right) k^{\alpha-l} \int_{\mathbb{R}} u^{\prime} \phi(u) d u \\
& =2^{\lrcorner \alpha}\left(k^{\prime}\right)^{\alpha} \sum_{l=0}^{\alpha}\left(\begin{array}{l}
\alpha \\
l
\end{array}\right)\left(k^{\prime}\right)^{-l} 2^{-l J} \int_{\mathbb{R}} u^{l} \phi(u) d u .
\end{aligned}
$$

The $2^{J \alpha}$ from the two inner products cancels out $h$, so for the right boundary functions we get

$M_{R}^{\alpha \beta}=\sum_{k=2^{J}-2 a+1}^{2^{\jmath}-1} \sum_{l=2^{J}-2 a+1}^{2^{J}-1} 2^{-J(\alpha+\beta)}\left\langle x^{\alpha}, \phi_{0, k}\right\rangle\left\langle x^{\beta}, \phi_{0, l}\right\rangle \int_{0}^{1} \phi_{J, k} \phi_{J, l} d x$.

Using this additional factorization results in significantly smaller condition numbers, but $M_{R}^{\alpha \beta}$ is still dependent on $J$ so we still have problems in some cases. The condition number of a square, non-singular matrix can be estimated as the ratio between the largest and smallest singular value $[21,(12.16)]$, therefore a singular value decomposition will result in three matrices where only the diagonal matrix with the singular values will have a condition number different from one. The matrix is given by $C_{R}=$ USV then $C_{R}^{-1}=V^{-1} S^{-1} U^{-1}$ and we can conclude that

$A_{R}=2^{-\frac{\jmath}{2}} V^{-1} S^{-1} U^{-1}$.

We will now consider the integral

$\int_{0}^{1} \phi_{J, k} \phi_{J, l} d x$

The integral can be calculated recursively, using the twoscale equation to split functions which are partially in the interval. This procedure is, however, computationally heavy. The integrals can be computed numerically by sampling $\phi_{j, k}$ and using a numerical integration method such as Simpson's rule.

We also wish to orthonormalize the Fourier-transformed boundary functions. In (21) we defined $M$ to be the inner product of the boundary functions. Due to Plancherel's theorem we have:

$M_{L}^{\alpha \beta}=\left\langle X_{J, \gamma^{\prime}}^{L}, X_{J, \eta}^{L}\right\rangle_{[0,1]}=\left\langle\hat{X}_{J, \gamma^{\prime}}^{L} \hat{X}_{J, \eta}^{L}\right\rangle_{[0,1]}$.

\section{The boundary wavelet package}

In Sect. 3 we derived the boundary functions mathematically. In this section we will describe the Python package and its use, [13]. A goal for the package is to be usable for a wide range of wavelets and applications.

\subsection{Package Overview}

The main functions are BoundaryWavelets and FourierBoundarywavelets which create the boundary functions and the Fourier transform of the boundary functions, respectively. The functions are dependent on NumPy and SciPy to run, [18]. To make best use of the package we also recommend using PyWavelets for its fast implementation of the cascade algorithm and its wide variety of wavelet coefficients. The accompanying test file depends on NumPy, SciPy, PyWavelets and Matplotlib.

The function BoundaryWavelets evaluates equation (10) for a given scale $J$ and $\alpha=\{0, \ldots, a-1\}$. This results in $2 a$ boundary functions. The evaluation is done using the rewriting of interior parts as described in Sect. 3 .

- Evaluate (10)

- Evaluate $\left\langle x^{\alpha}, \phi_{J, k}\right\rangle$ according to (12)

- Evaluate $\left\langle x^{\prime}, \phi\right\rangle$ according to (14)

Similarly, the function FourierBoundarywave$l$ ets evaluates equation (15) for a given scale $J$ and $\alpha=\{0, \ldots, a-1\}$. This also results in $2 a$ boundary functions, but involves a few additional steps.

- Evaluate (15)

- Evaluate $\left\langle x^{\alpha}, \phi_{J, k}\right\rangle$ according to (12)

- Evaluate $\left\langle x^{\prime}, \phi\right\rangle$ according to (14)

- Find the Fourier transform of $\phi$ on the interval according to (16)

- Evaluate the Fourier transform of $\phi$ using (17)

- Evaluate the Fourier transform of the window using (18)

\subsection{Using the package}

The most important thing when using the package is giving the functions suitable inputs.

BoundaryWavelets (phi, J, WaveletCoef, A $\mathrm{L}=\mathrm{None}, \mathrm{AR}=\mathrm{None}$ ) takes five inputs, where two are optional. Firstly phi is the interior scaling function for the wavelet. It can be found using the cascade algorithm, the number of samples in phi dictates the number of samples in the boundary functions in the scale:

\#samples $\left(\phi_{L}\right)=\frac{\# \text { samples }(\phi)}{2 a-1}$.

$J$ is the scale, it must be chosen such that $\operatorname{supp}\left(\phi_{J, 0}\right) \subseteq[0,1]$, for Daubechies wavelets $J \geq a$. The 


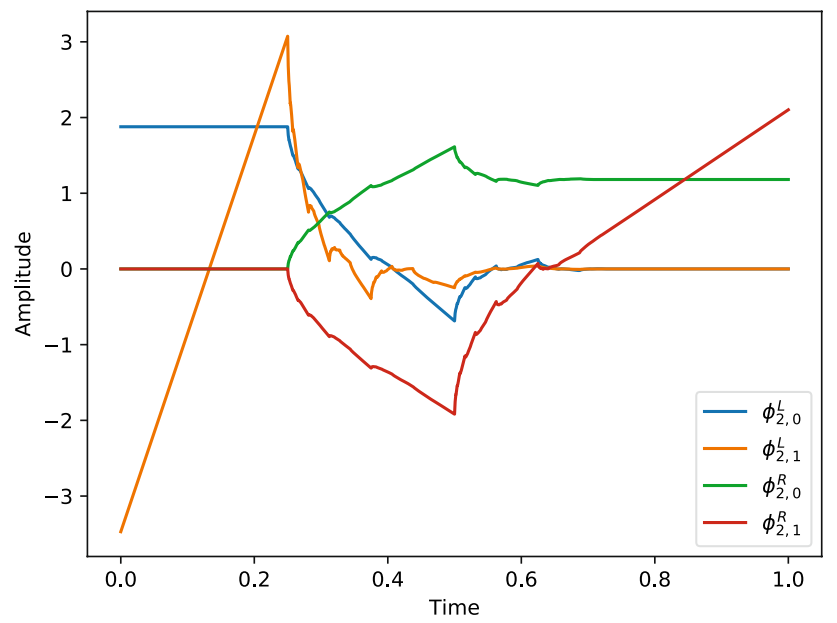

Fig. 1 Daubechies 2 boundary wavelet scaling functions

Wave letCoef parameter is the wavelet coefficients, note that if the PyWavelets package is used to find these, their ordering must be reversed. We refer to [7, Table 6.1] for Daubechies wavelet coefficients. The last two parameters $A L$ and $A R$ are the left and right orthonormalization matrices. They can be created using the function OrthoMatrix. If they are not given, the boundary functions will not be orthonormalized.

FourierBoundaryWavelets ( $\mathrm{J}$, Scheme, wave letCoef, $\mathrm{AL}=$ None, $\mathrm{AR}=$ None, Win=Rectangle) takes six inputs, where three are optional. J, WaveletCoef, AL and AR are as before. Scheme is the sampling scheme i.e. an array of the frequencies in which to sample. The parameter $W$ in is Rectangle as standard. The Fourier transform of $\phi_{J, k}$ is convolved with this function in order to limit it to the interval $[0,1]$ as in (16). Alternative window functions are discussed in Sect. 8.

\section{Example}

In this section we derive boundary wavelet scaling functions for the Daubechies wavelet with two vanishing moments. In this case we need two left and two right functions.

We use the test function WaveletTest. Generaltest ( TimeOnly=True) to plot the boundary functions, see Fig. 1. The Boundary functions are supported on the same interval as the outermost interior function. Here $J=2$ and the orthonormalization matrices are

$$
A_{L}=\left(\begin{array}{cc}
1.8781 & 0 \\
-16.562 & 26.182
\end{array}\right), \quad A_{R}=\left(\begin{array}{cc}
1.1814 & 0 \\
-6.7903 & 5.9285
\end{array}\right) \text {. }
$$

Table 1 Table of coefficients for the functions $f$ and $g$

\begin{tabular}{lll}
\hline & $f(x)=1$ & $g(x)=x+0.366$ \\
\hline$\alpha_{0}$ & 0.37649458 & 0.33743841 \\
$\alpha_{1}$ & 0 & 0.10802637 \\
$\alpha_{2}$ & 1 & 2 \\
$\alpha_{3}$ & 1 & 3 \\
$\alpha_{4}$ & 1 & 4 \\
$\alpha_{5}$ & 1 & 5 \\
$\alpha_{6}$ & 0.59851575 & 4.15814292 \\
$\alpha_{7}$ & 0 & 0.47705625 \\
\hline
\end{tabular}

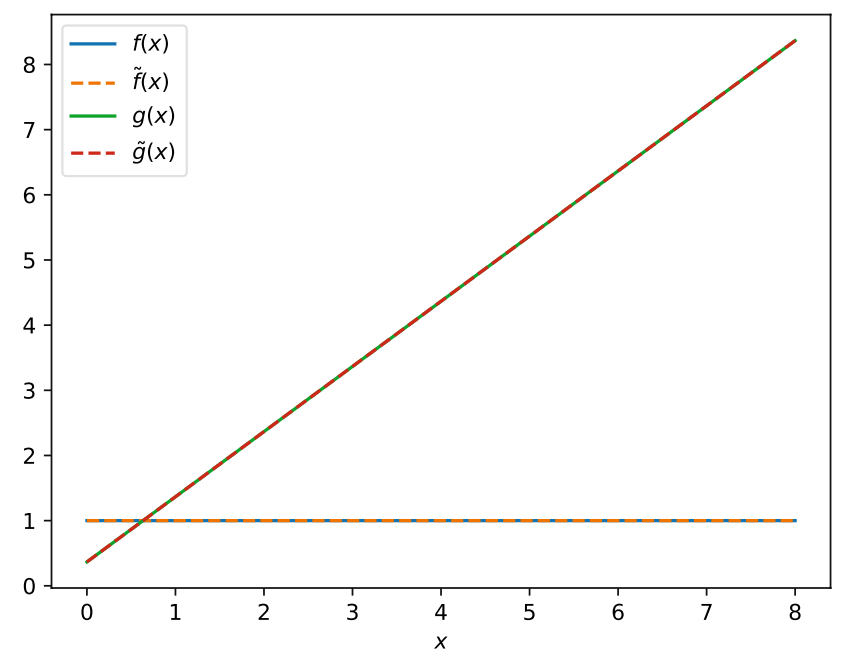

Fig. 2 The functions $f$ and $g$ and their reconstructions

\subsection{Test}

To check that we are able to reconstruct polynomials up to degree 1, we choose two functions: a constant function and a first degree polynomial. We try to find coefficients which give perfect reconstruction. For both cases we use a scale of three, i.e. $J=3$, and use the orthonormal boundary functions.

The functions chosen are $f=1$ and $g=x+0.366$. All of the coefficients have been found using the test function Recontest.TestofConfunc () and are written in Table 1. The reconstructions using these two sets of coefficients are shown in Fig. 2. It is visually evident that the reconstructions are very good. Furthermore, the distance between the true signals and the reconstructed ones are $\|f(x)-\tilde{f}(x)\|_{2}=3.39 \cdot 10^{-13}$ and $\|g(x)-\tilde{g}(x)\|_{2}=4.41 \cdot 10^{-11}$. 


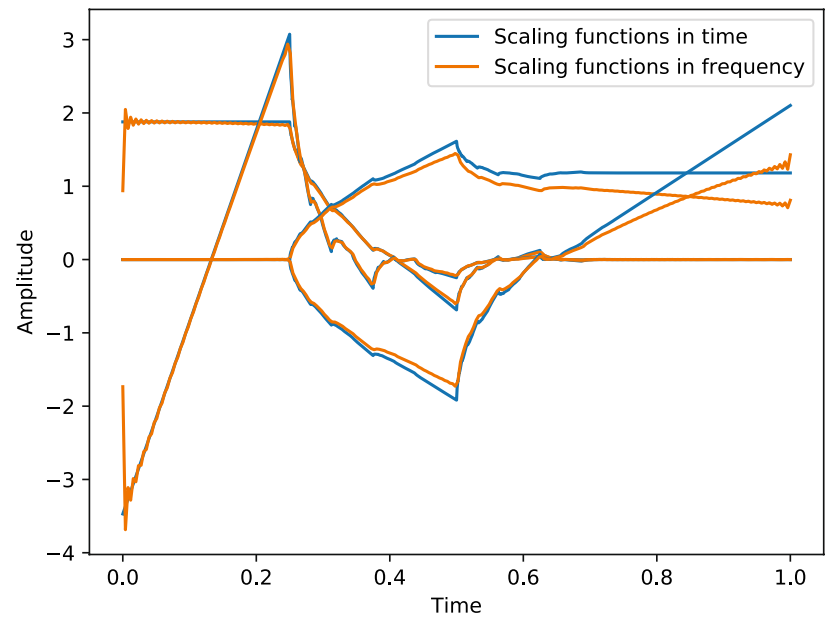

Fig. 3 Comparison of boundary functions created in the time(blue) and the frequency (orange) domain

\subsection{The frequency domain}

The boundary wavelet scaling functions have been constructed in the frequency domain sampled uniformly in the interval $[-128,128)$ with a density of $\frac{1}{7}$ and scale $J=2$. This is done using the test function WaveletTest. Generaltest (). We can compare these functions to the boundary functions in time using a discrete inverse Fourier transform and the relation

$f(x)=\epsilon^{-\frac{1}{2}} \mathcal{F}^{-1}\left[\epsilon^{\frac{1}{2}} \hat{f}(\epsilon \omega)\right]\left(\frac{1}{\epsilon} x\right)$.

This comparison can be seen in Fig. 3 .

It is visually evident, in Fig. 3, that the boundary wavelet scaling functions from the frequency domain are similar to functions created in the time domain. Due to the convolution with the Fourier transformed indicator function and the limited sampling interval in the frequency domain there is some inaccuracy and Gibbs phenomenon. These effects cannot be avoided when the functions have compact support in the time domain and are discontinuous at the boundary.

\section{Comparison to classical wavelet approach}

In this section we compare wavelet decomposition and reconstruction using our wavelet basis to a classical approach using a mirrored extension of the signal. With mirrored extension we approximate the signal outside the known interval by a mirrored version of itself. We have used real-life ECG data for the test as this type of data is well represented by wavelets, [22]. We have used
Table 2 Results of the tests. The table contains the minimum, maximum and mean of the error for both methods.

\begin{tabular}{lcc}
\hline Test 1 & Boundary functions & Mirror extension \\
\hline Minimum & 0.81 & 0.32 \\
Maximum & 6.88 & 32.95 \\
Mean & 1.86 & 2.93 \\
Test 2 & & \\
Minimum & 0.66 & 0.38 \\
Maximum & 12.78 & 43.50 \\
Mean & 2.55 & 3.72 \\
Test 3 & & \\
Minimum & 0.67 & 0.41 \\
Maximum & 18.31 & 54.21 \\
Mean & 2.13 & 6.15 \\
\hline
\end{tabular}

the dataset Combined measurement of ECG, breathing and seismocardiogram ${ }^{1}$ described in $[8,9]$ for the test. The data set is available in PhysioNet [11]. This dataset contains four rows of data, the test will be done on the first two (the ECG signal channels).

We have chosen to use 12 steps of the cascade algorithm to sample the basis functions and therefore we need test signals of length $2^{12}$. With the chosen dataset we can make 332 disjoint test signals where the samples in each signal is the interval $\left[n 2^{12},(n+1) 2^{12}\right)$. Furthermore we have chosen to use the Daubechies 3 and 5 wavelets at a scale of 7. For the wavelet basis with boundary functions this choice of scale results in 128 coefficients. Due to the special construction of the boundary functions we need $2 a-2$ extra coefficients to represent the signal using the mirrored extension method.

The error has been calculated for each of the 332 test signals as

$\frac{\|f-\tilde{f}\|_{2}}{\|f\|_{2}}$.

The test has been done using DataTest. Test (), the minimum, maximum, and mean of these errors are shown in Table 2. Test 1 and 2 are Daubechies 3, and test 3 is Daubechies 5. The test shows that overall the wavelet basis with boundary functions performs better.

Figures 4 and 5 show examples of a section where the difference between the error for the two reconstructions is largest, it can be reproduced using the function DataTest. TestPlot (). Furthermore, the test shows that for most signals the reconstruction using mirrored extension is slightly better. However, for some types of signals the

\footnotetext{
1 https://doi.org/10.13026/C2KW23.
} 


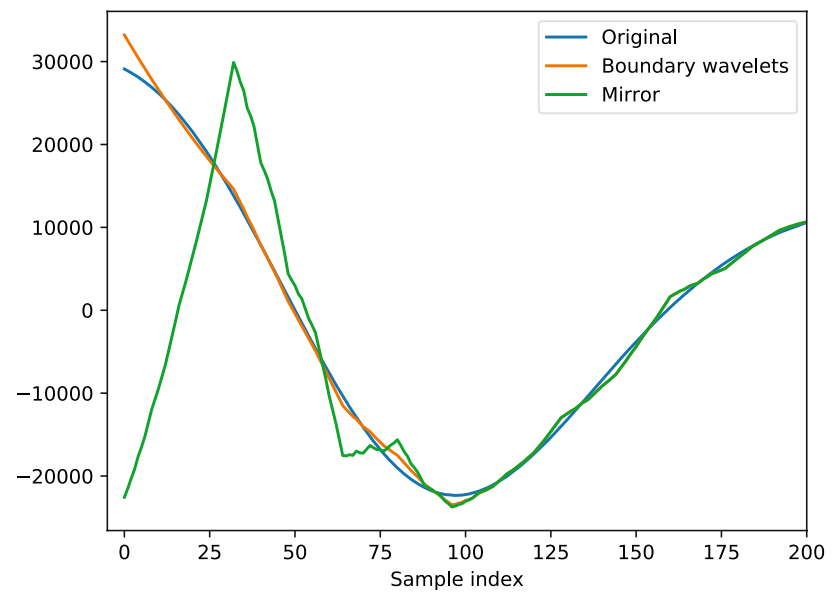

Fig. 4 Example of reconstruction using boundary functions vs. mirrored extension using Daubechies 3

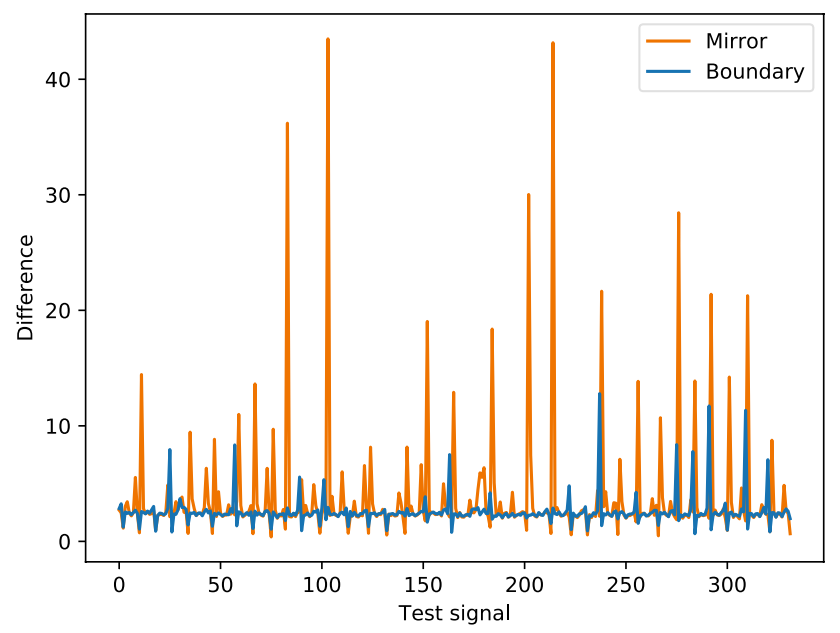

Fig. 5 Comparison of error for the two methods using Daubechies 3

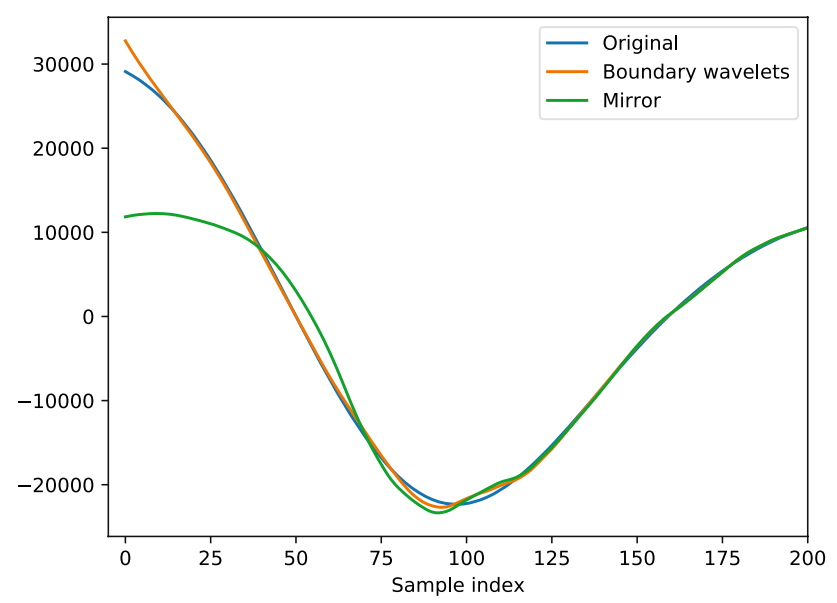

Fig. 6 Example of reconstruction using boundary functions vs. mirrored extension using Daubechies 5

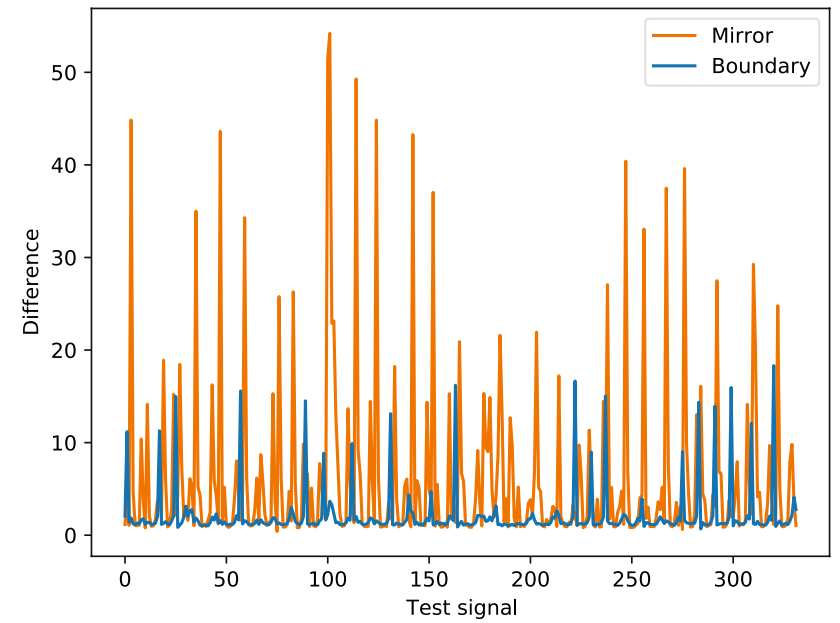

Fig. 7 Comparison of error for the two methods using Daubechies 5

mirrored extension method commits a large error. Figure 4 is an example of such a signal, the characteristic being the steep downwards slope at the start. The reconstruction with boundary functions on the other hand does make errors but not as large as the mirrored extension. Figures 6 and 7 show the difference between the original and the two reconstructions for each signal. In most of the cases where the boundary function method commits larger error than its average, the mirrored extension method commits a similar error, which suggests that the major part of the error is not committed at the boundary.

\section{Discussion}

In this paper we have described the explicit construction of boundary wavelet scaling functions and their Fourier transform, with the purpose of generalized sampling in mind. These boundary functions are moment-preserving by design and when using the carefully constructed orthonormalisation matrix they are also orthonormal. This construction of orthonormal, moment-preserving boundary functions has been implemented in Python and we have shown that it has the desired properties. Compared to other implementations of boundary wavelet scaling functions such as $[10,14]$, which rely on boundary filter coefficients and are therefore limited to the wavelets for which such coefficients have been calculated, our work relies on the filter coefficients of the interior wavelets. Therefore our method and implementation can be used for a wider range of wavelets. When comparing the decomposition and reconstruction, of real world ECG data, using our boundary functions, to the classical approach of a mirrored extension of the signal at the boundary, we 
found that boundary functions are better on average. The mirrored extension approach is generally good, but for some types of boundary behaviour it commits large errors. The boundary function approach is more stable and less dependent on the signal's behaviour.

\section{Conclusion}

In Sect. 3.1 the Fourier transform of $\phi$ is convolved with the Fourier transform of the indicator function in order to restrict it to the interval $[0,1]$ in time. In a way the indicator function works as a window in this setting and it is therefore appropriate to discuss other possible windows. The indicator function is the ideal window in time, but its discontinuous edges means that its Fourier transform has very slow decay. When using a window function there is always a trade-off between the damping of the function in time and the rate of decay of its Fourier transform. The window function is an optional input to FourierBoundaryWavelets, so choosing the best window function for a given problem is up to the user. The computational complexity of the construction of the boundary functions is relatively high, but it is important to note that it is a one time expense. Once the boundary functions have been evaluated they can be saved and used over and over at a similar cost to the interior basis functions. The use of the proposed boundary functions as part of a wavelet transform can easily be extended to more dimensions, [12]. First, if the signal is not already discrete, sample it on a regular grid. For a 2D signal apply the transform described in Sect. 6 to all rows and all columns of the signal. In general, an $\mathrm{N}$-dimensional signal can be split into 1D elements $N$ different ways, so the transform can be applied to the elements one dimension at a time. One important reason for expanding to more dimensions is the use in the field of MRI, since MR images are 3D. The current paper is meant as a toolbox for researchers interested in applying wavelets on an interval for many different purposes. We show an example of the usefulness of the boundary functions for compression, but the functions could also be useful for other areas such as interpolation and noise reduction.

Funding The department of Mathematical Sciences, Aalborg University.

Availability of data and material and Code availability GitHub: https ://github.com/JosefineAtMath/BoundaryWavelets

\section{Compliance with ethical standards}

Conflict of interest On behalf of all authors, the corresponding author states that there is no conflict of interest.

\section{References}

1. Adcock B, Hansen AC (2012) A generalized sampling theorem for stable reconstructions in arbitrary bases. J Fourier Anal Appl 18(4):685-716

2. Adcock B, Hansen AC, Poon C (2014) On optimal wavelet reconstructions from fourier samples: linearity and universality of the stable sampling rate. Appl Comput Harmonic Anal 36(3):387-415

3. Andersson L, Hall N, Jawerth B, Peters G (1994) Wavelets on closed subsets of the real line. $\ln 21$

4. Christensen $\mathrm{O}$ (2008) Frames and Bases, an introductory course, 1 st edn. Birkhäuser, Basel

5. Cohen A, Daubechies I, Jawerth B, Vial P (1993) Multiresolution analysis, wavelets and fast algorithms on an interval. Comptes Rendus de l'Académie des Sciences. Série I 316

6. Cohen A, Daubechies I, Vial P (1993) Wavelets on the interval and fast wavelet transforms. Appl Comput Harmonic Anal 1(1):54-81

7. Daubechies I (1992) Ten lectures on wavelets, vol 61. Siam, New Delhi

8. García-González M, Argelagós-Palau A, Fernández-Chimeno M, Ramos-Castro J (2013) Differences in qrs locations due to ecg lead: relationship with breathing. IFMBE Proc 41:962-964

9. García-González MA, Argelagós-Palau A, Fernández-Chimeno M Ramos-Castro J (2013) A comparison of heartbeat detectors for the seismocardiogram. In: Computing in Cardiology Conference (CinC)

10. Gataric M, Poon C (2016) A practical guide to the recovery of wavelet coefficients from fourier measurements. SIAM J Sci Comput 38(2):A1075-A1099

11. Goldberger AL, Amaral LAN, Glass L, Hausdorff JM, Ivanov PC, Mark RG, Mietus JE, Moody GB, Peng CK, Peng Chung-Kang a Stanley HEPHEP (2000) Physiobank, physiotoolkit, and physionet: Components of a new research resource for complex physiologic Physiobank, physiotoolkit, and physionet: components of a new research resource for complex physiologic signals. Circulation 101(23):214-220

12. Hansen $A C$, Thesing $L$ (2020) On the stable sampling rate for binary measurements and wavelet reconstruction. Appl Comput Harmonic Anal 48(2):630-654

13. Holm J, Nielsen SL (2019) Boundarywavelets python package. https://github.com/JosefineAtMath/BoundaryWavelets/tree/1.0

14. Jacobsen RD, Nielsen M, Rasmussen MG (2017) Generalized sampling in julia. J Open Res Softw 5(1):12

15. Jawerth B, Sweldens W (1994) An overview of wavelet based multiresolution analyses. SIAM Rev 36(3):377-412

16. Kessler B, Payne G, Polyzou W (2003) Wavelet notes. arXiv:nucl-th/0305025

17. Mallat S (2009) A wavelet tour of signal processing, 3rd edn. Elsevier, Amsterdam

18. Oliphant TE (2007) Python for scientific computing. Comput Sci Eng 9(3):10-20

19. Ruch DK, Van Fleet PJ (2011) Wavelet theory: an elementary approach with applications. Wiley, New York

20. Strang G, Nguyen T (1996) Wavelets and filter banks. SIAM, New Delhi

21. Trefethen LN, Bau D III (1997) Numerical linear algebra, vol 50. Siam, New Delhi

22. Unser M, Aldroubi A (1996) A review of wavelets in biomedical applications. Proc IEEE 84(4):626-638

23. Wickerhauser MV (1996) Adapted wavelet analysis: from theory to software. AK Peters/CRC Press, Natick

Publisher's Note Springer Nature remains neutral with regard to jurisdictional claims in published maps and institutional affiliations. 